Artículo

Formación docente

revista iberoamericana de educación

\title{
Representaciones sociales de masculinidad, un proceso de identidad del hombre
}

Social representations of masculinity, a man's identity process

Representações sociais da masculinidade, processo de identidade do homem

\section{Darwin Alexis - Cruz García}

Corporación Universitaria Minuto De Dios- Uniminuto Trabajador social, magister en Estudios políticos, perteneciente grupo Colciencias Nexos: Narrativas y experiencias de organizaciones y sujetos - Colombia. Email. Darwin.cruz@uniminuto.edu.co ORCID: https://orcid.org/0000-0002-1858-1945

\section{Diana Carolina Tibaná Ríos}

Docente investigadora del programa de trabajo social de la Corporación Universitaria Minuto de Dios. Trabajadora Social graduada de la Universidad de la Salle y magíster en desarrollo educativo y social de la universidad pedagógica nacional. Integrante del grupo de investigación reconocido por COLCIENCIAS "conocimiento en contexto". Líder del semillero de investigación Social Waves en el cual se desarrollan las líneas de trabajo relacionadas con el trabajo social digital. Dtibana75@unisalle.edu.co.

ORCID: https://orcid.org/0000-0003-2732-3603

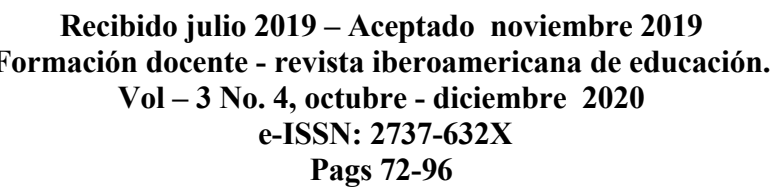

Resumen El estudio de las representaciones sociales de lo masculino es algo importante hoy en día ya que plantea la reflexión en la construcción de identidad del hombre, siendo el eje en la construcción de nuevas formar de relación y de percepción de lo masculino cuestionando las posturas hegemónicas. Se presenta un estudio descriptivo de los procesos de identidad que tiene la población de la comuna 2 de Soacha de los hombres en el marco de las nuevas masculinidades, participaron 14 instituciones y se recogieron 292 encuestas, 177 de mujeres y 115 de hombres. Por tanto, los resultados en las características en el proceso de identidad del hombre se plantean a través de procesos de socialización que por medio del lenguaje imponen colores, forma de vestir y roles. Finalmente, las representaciones sociales comprenden la masculinidad desde enunciados y códigos lingüísticos, en 
estructuras familiares y sociales relacionados a modelos culturales dominantes.

Palabras clave: Familia, identidad, masculinidad, representación social.

\begin{abstract}
The study of the social representations of the masculine is something important today since it raises the reflection on the construction of man's identity, being the axis in the construction of new forms of relationship and perception of the masculine, questioning hegemonic positions. A descriptive study of the identity processes that the population of the commune 2 of Soacha has of men in the framework of the new masculinities, 14 institutions participated and 292 surveys were collected, 177 from women and 115 from men. Therefore, the results in the characteristics in the man's identity process are raised through socialization processes that through language impose colors, dress and roles. Finally, social representations understand masculinity from linguistic statements and codes, in family and social structures related to dominant cultural models.
\end{abstract}

Keywords: Family, identity, masculinity, social representation.

Resumo: O estudo das representações sociais do masculino é algo importante na atualidade, pois suscita a reflexão sobre a construção da identidade do homem, sendo o eixo na construção de novas formas de relação e percepção do masculino, questionando posições hegemônicas. Num estudo descritivo dos processos identitários que a população da comuna 2 de Soacha tem de homens no quadro das novas masculinidades, participaram 14 instituições e recolheram-se 292 inquéritos, 177 de mulheres e 115 de homens. Portanto, os resultados nas características do processo de identidade do homem são elevados por meio de processos de socialização que por meio da linguagem 
impõem cores, vestimentas e papéis. Finalmente, as representações sociais entendem a masculinidade a partir de enunciados e códigos linguísticos, em estruturas familiares e sociais relacionadas aos modelos culturais dominantes. Palavras-chave: Família, identidade, masculinidade, representação social.

Palavras-chave: Teoria Crítica, Pensamento Crítico, Subjetividades anticapitalistas, Zapatismo, Uthopia.

\section{INTRODUCCIÓN}

Hablar de género en la actualidad se ha vuelto un tema controversial dentro de la sociedad no solo por la infinidad de movimientos que han ido apareciendo con el pasar de los años (Lopes, 2019), sino por los cambios de ideales que defienden estereotipos sociales del ser hombre en la actualidad siendo así un proceso de cualificar lo masculino (Tubay, 2019). Entonces, la necesidad del mundo contemporáneo en el hombre plantea la necesidad en la comprensión de la evolución de su papel en la sociedad (Del Río \& Pastrana, 2018).

Esto fundamenta la crisis de la masculinidad contemporánea relacionado a cambios sociales fundamentados en movimientos feministas que debaten los roles de acuerdo al género en la sociedad (Romero \& Gea, 2019). Por esta razón, es importante generar un acercamiento frente a las discusiones actuales de masculinidad que permita identificar esas características en las relaciones de poder que transcienden en el rompimiento de estereotipos hegemónicos (Albelda, 2018).

De este modo, comprender lo masculino como aquellas construcciones sociales asociadas a funciones, comportamientos y actividades que se reproducen en la sociedad plantea la necesidad en el cuestionamiento en la construcción de la identidad del hombre y la masculinidad (Viveros, 2018). Estas plantean funciones y comportamientos asociadas al género que crean 
desigualdad y el desarrollo de nuevas percepciones en lo personal, familiar y social (Torns \& Recio, 2012).

Entonces, comprender las nuevas formas de masculinidad en relación al análisis del género como categoría de movimientos feministas es cada vez más frecuente en las ciencias sociales para dar cuenta de los cambios en los últimos tiempos del papel del hombre (Bedia, 2005). Es decir, que todo aquello que antiguamente se consideraba propio de los hombres o mujeres en vestimenta, actitudes, gestos y funciones sociales considerados correctos han ido cambiando (Connel \& Pearse, 2018).

Las representaciones sociales del hombre se han transformado reconstruyendo poco a poco la forma de entender el género a partir de lo tradicionalmente aceptado desarrollando reflexiones para pensar lo masculino en la sociedad actual a partir del ajuste en su papel y proyección de calidad de vida (Gomes, 2018). Esto plantea el rompimiento de prejuicios sociales sobre el hombre que influyen y generan conflicto en lo personal, familiar y social, debido al no cumplimiento de estereotipos asociados de proveedor, protector o líder de procesos en la sociedad actual (Donat \& Díaz, 2013).

De este modo, las reflexiones sobre la masculinidad plantean las diferencias del género más allá de lo observable entre el ser hombre o mujer en la sociedad, por ejemplo en en el diseño de las políticas públicas que tienen como pretensión ser coherentes a las necesidades de estereotipos sociales del género (Bard, 2018). Por tanto, se estructuran programas que quieren optimizar los servicios y dar soporte a las necesidades de una sociedad, pero no a las necesidades de los hombres y a los cambios sociales que han desarrollado formas diferentes de identidad de hombres y mujeres (Bautista \& Barquín, 2018).

Así, el patriarcado ha creado un sistema social y cultural a partir de la figura masculina, pero no de cualquier figura masculina, sino del hombre rico, 
blanco, nacido en el primer mundo, con un grado alto de educación recibida en escuelas de occidente y heterosexual (Muñoz, 2018). Ciñéndonos estrictamente a este modelo, se sustenta la reflexión frente a ¿Cuáles son las Representaciones sociales de masculinidad que tiene la población de la comuna 2 de Soacha frente al proceso de identidad del hombre?

Esto plantea la necesidad de conceptualizar la percepción del hombre más allá del sujeto fuerte y de poder que se forma en el contexto familiar (Gallego, 2018). Planteando la reflexión en la manera en cómo se construye la identidad del hombre de manera sistemática en el medio ambiente, la familia, la comunidad, que permite evidenciar patrones que dan respuestas a las necesidades de los hombres en la relación salud-enfermedad, social, económico, cultural (Cabezas, 2019).

Sin embargo, en el momento de implementar los diferentes modelos de comprensión del hombre en un contexto como la familia, se visibiliza incoherencia con la realidad soportada en problemas de manejo de la salud, seguridad, trabajo y (Montenegro \& Otros, 2019). Esto plantea en las acciones del Estado la implementación de modelos que no son acordes a las necesidades del hombre y a las realidades sociales contemporáneas (Bard, 2018).

En este sentido, es importante analizar los imaginarios de identidad que tienen los hombres, en la dimensión del rol en el contexto familiar y social, ya que puede generar una identidad social y un capital humano diferente a la percepción tradicional (Viveros, 2018). En donde, la capacidad de vincularse a las dinámicas del mercado, a partir de las políticas públicas, estas entendidas como la suma de las actividades del gobierno bien sea directamente o a través de agentes, las cuales tienen influencia en la vida de los ciudadanos (Bautista \& Barquín, 2018). 
De este modo, la presente investigación de carácter descriptivo avanza en la construcción de una identidad de los hombres en el municipio de Soacha; a partir, de la dinámica entre lo biológico, el rol social y lo político - cultural en el contexto de la construcción de imaginarios alrededor de ser hombre (Núñez \& Espinoza, 2017). Esto plantea una interpretación del hombre desde lo biológico en el reconocimiento de sus roles y el proceso de una construcción de identidad individual y colectiva, en el manejo de toma de decisiones, en la motivación en la participación en espacios institucionales (Foraster \& Folch, 2019).

En donde la familia, la escuela y demás entornos de formación del ser humano, se ha precisado una única forma de ser hombre de acuerdo a la tradición cultural de los países occidentales, que mantienen una religiosidad primordialmente católica o cristiana, y cuyo modelo educativo privilegia a los varones por encima de las mujeres, a los ricos sobre los pobres, a los blancos sobre las personas de diferentes comunidades étnicas y a los heterosexuales sobre las personas sexualmente diversas (Moreno, 2017).

Por tanto, se puede plantear que quizás los hombres se perciben en la historia como verdugos y victimarios en la concentración de poder que manifiesta relaciones de dominación (Cabezas, 2019). Es decir, su papel de verdugos está acentuado porque parece que vivir con su masculinidad, fuera un decreto descubriendo el rol social, los comportamientos, prácticas, hábitos, estilos de vida, pautas de crianza y estrategia de empoderamiento que quizás argumentan una nueva percepción del ser hombre (Moreno, 2017).

Finalmente, la masculinidad hegemónica acentúa en los hombres los rasgos de competitividad y de violencia, al mismo tiempo que se concentra en un ejercicio constante del poder masculino, esto hace que los hombres, en muchas oportunidades se vean obligados desde su identidad a un ejercicio recurrente de la fuerza (Gómez, 2019). Esto para dar respuestas a expectativas 
sociales asociadas al no poder escapar de su identidad, no poder llorar, no poder sentir, estar siempre dispuestos al sexo o a la disputa, estar sujetos siempre a la mirada crítica de la sociedad, lo cual en muchas oportunidades pone en riesgo su vida y su salud (Martínez, 2019).

Por tal motivo, este procedimiento investigativo es de gran utilidad e importancia, porque posibilita dan un soporte teórico desde la identificación de una identidad para el hombre, posibilitando una construcción colectiva del concepto masculino (Pineda \& Otros). Por tanto, plantea nuevas formas de relaciones de equidad con relación al desarrollo social, respetando las condiciones individuales y la diversidad que contiene el territorio en la percepción de instituciones públicas y en el género (Bautista \& Barquín, 2018).

\section{MATERIALES Y MÉTODOS}

El presente estudio fue realizado durante el 2019 en el municipio de Soacha, en el marco de la investigación imaginarios identitarios que tiene la población de la comuna 2 frente a los roles del hombre en el contexto familiar y social. El interés del estudio es describir la situación de los procesos de identidad del hombre con respecto a las nuevas masculinidades. Para ello, se realizó una aproximación a estas representaciones sociales por medio de un cuestionario, a partir de la información recolectada se profundiza en determinantes conceptuales por medio de grupos focales.

De este modo, se profundiza en las temáticas destaca los imaginarios de identidad sobre masculinidad, pues es un punto que emerge con potencia en las respuestas abiertas. Por tanto el objeto d estudio de este artículo es describir los procesos de identidad que tiene la población de la comuna 2 de Soacha de los hombres en el marco de las nuevas masculinidades, y plantear la necesidad en la reflexión de esos nuevos roles sociales. 
Se desarrolló un cuestionario a población general de la comuna 2 de Soacha creado a partir del proceso de categorización y de carácter deductivo, para identificar aspectos en relación a las nuevas masculinidades (Newman, 2006). Con este instrumento se plantea un acercamiento a la realidad social de la comuna 2, por medio de los imaginarios y representaciones sociales del hombre, por esto las preguntas eran de carácter cerrado para la elección de una opción, frecuencia o prioridad. En consecuencia, se desarrolla un análisis a estas respuestas y junto a las entrevistas semiestructuradas desarrolladas a un grupo de hombres seleccionados ad hoc, consolidan las categorías emergentes para elaborar los grupos focales, formados por 10 personas funcionarios institucionales, líderes comunitarios, hombres y mujeres del sector.

Por tanto los grupos focales, tuvieron una duración de 45 minutos, los ejes guía hacían referencia a los diferentes roles del hombre en el marco de las nuevas masculinidades $\mathrm{y}$ en los estereotipos hegemónicos socialmente aceptados, a la familia como agente de conocimiento y difusión de patrones de género, así como a la identidad entendida como una construcción social y cultural.

Participaron 14 instituciones y se recogieron 292 encuestas, 177 de mujeres y 115 de hombres. La edad media de quienes han respondido es de 32,36 años y la media de años de experiencia de funcionarios institucionales es de 12, 05 años. En los grupos focales participaron 8 mujeres y 16 hombres de la comuna 2 de Soacha, 3 mujeres y 5 hombres líderes comunitarios de la comuna y 7 mujeres y 3 hombres funcionarios de las diferentes instituciones. 2.4 Análisis de la información

El análisis e la información se fundamentan en la información cuantitativa, ya que el interés de a investigación se centraba en describir los imaginarios frente a la construcción de la identidad del hombre en el marco de las nuevas 
masculinidades en la comuna 2 de Soacha. De este modo, las respuestas cualitativas recogidas de las transcripciones de las entrevistas y grupos focales se introdujeron en el programa Nvivo-plus, que con enfoque hermenéutico posibilita la comprensión de los datos a partir de indagar situaciones en las relaciones del contexto en particularidades, simbologías, imaginarios, significados, percepciones, narrativas (Pérez \& Radi, 2018). Por otra parte, con los datos cuantitativos se realizó un análisis de frecuencias, para identificar a los hombres que hacen parte de las entrevistas, y aportan información para contrastar con los datos cualitativos recolectados. Sin embargo, es importante señalar que estos no son prioritarios para la investigación por tanto no son significativos desde el punto de vista estadístico.

\section{RESULTADOS}

La identidad sexual y de género de las personas atraviesa un debate interesante en el que constantemente se abren un mayor número de categorías, para poder definir especialmente desde aspectos sociales a los seres humanos (Sánchez, 1988). De este modo, la información que proporcionan las personas señala que la identidad en medida se fundamenta en un 55\% a lo biológico, un $30 \%$ a la expresión y un $20 \%$ lo relaciona con la orientación. Por tanto, esta situación en la construcción de la identidad soporta la tesis de la fragmentación de la persona desde diferentes perspectivas que dificulta un abordaje integral, pero sustenta una atención diferencial en el marco institucional (Alcántara, 2013).

En donde, los funcionarios institucionales indican que la identidad de género se ubica en la influencia de la cultura a partir de los roles, funciones o estatus del hombre en el contexto familiar (Izquierdo, 2010). De este modo, se consolida que la expresión de género en el marco social y comunitario de la 
comuna 2 de Soacha se fundamenta en roles del hombre impuestos tradicionalmente; en donde, las actividades que se relacionan al proceso de identidad del hombre está en un $86 \%$ relacionada a la productividad y un $14 \%$ lo relacionan en actividades del hogar (Lagarde, 2018).

Entonces, la identidad del hombre como expresión de género predominantemente es impuesta a partir de sus formas biológicas (Lama, 2013). No obstante, también se contrasta con la percepción de los jóvenes que son el $45 \%$ de los sujetos que participaron del estudio señalan que la identidad se construye desde la autonomía personal basada en las herramientas culturales del entorno (Butler, 2004). En esta medida, las personas se pueden encontrar en una ambivalencia frente a su identidad de género que va entre lo que le ha sido impuesto y su posibilidad de construcción personal.

Por tanto, los resultados en las características en el proceso de identidad del hombre se plantean en escenarios como la familia que orienta la forma de comportamiento del hombre a través de procesos de socialización que por medio del lenguaje imponen colores, forma de vestir y roles (Salinas \& Núñez, 2019). De este modo, las nuevas formas de construcción de la identidad del hombre se relacionan a través de nuevas estructuras y relaciones sociales ligadas a una transformación sustancial y profunda de los patrones culturales dominantes soportadas en la forma como se mira, lee, interpreta y actúa el hombre (Botero, Montoya \& Opina, 2019).

Finalmente, el proceso de identidad se plantea en condiciones biológicas y genéticas que vincula estructuras mentales de como el otro reconoce e interactúa cotidianamente con el hombre, que presupone relaciones a partir de modelos estereotipados sustentados en patrones culturales (Rubinacci, 2019). En consecuencia, la identidad se puede comprender en el hombre a través del rompimiento de paradigmas tradicionales y en la necesidad de 
estudiar y comprender formas nuevas desde lo simbólico, el poder y roles sociales (Cruz \& Ospina, 2020).

El conocimiento de las representaciones sociales como procesos de imágenes mentales y expresión de conocimientos frente a lo masculino orienta comportamientos de los sujetos de estudio en un 53\% asociado a formas de comunicación, el $42 \%$ al lenguaje y un $5 \%$ a la clonación de comportamientos asociados a la repetir acciones del abuelo o padre. Es decir, toman la actitud de sus acciones proporcionado a los hombres medios para guiarse en lo social y material (Winfield, Jiménes \& Topete, 2017).

La categoría de representaciones constituye un elemento teórico indispensable para la reflexión sobre los procesos de identidad del hombre y la masculinidad vinculados a la mediación de la comunicación que sumen posturas culturales frente a las relaciones de poder (Martel, 2017). Esta postura plantea en los hombres una situación que expresa dominio y la poca posibilidad de liberarse de las limitaciones del paradigma hegemónico de la masculinidad, cómo resultado de la relación del Estado que reduce al hombre en actitudes y limita la percepción a nuevas formas de masculinidad que se manifiestan en el contexto (Rivera \& De los Santos, 2017).

Entonces, aceptar la masculinidad inherente al concepto de representación desde el tejido social constituido para aceptar al otro a partir de relaciones de poder plantea la mediación del pensamiento y la conducta del hombre asociado la percepción de su rol social (Moncayo \& Rodríguez, 2019). En donde, los sujetos de estudio comienzan a comprender la masculinidad desde enunciados y códigos en un $40 \%$ lingüísticos, un $35 \%$ en estructuras familiares $\mathrm{y}$ sociales, $\mathrm{y}$ un $25 \%$ lo relacionan a modelos culturales dominantes.

Por tanto, comprender las relaciones sociales como método para asemejar la realidad y desarrollar pensamientos que conllevan a una acción de los 
hombres como agentes de una masculinidad hegemónica plantea retos en la construcción de símbolos para nuevos significados de la masculinidad (Martínez \& Otros, 2018). De este modo el papel de la familia es importante para constituir las conductas y funciones de los hombres en función a la masculinidad y la transmisión de valores sociales.

Finalmente, se evidencia cambios respecto a las representaciones sociales de los hombres relacionados a los roles dentro de la familia, a pautas de crianza de los niños y a la necesidad de la inmersión de la mujer en lo laboral, aspectos que transcienden la mirada tradicional de la masculinidad (Mio ,2018). En consecuencia, las representaciones sociales de la masculinidad son reproducidas en el contexto social a partir de la comprensión en relaciones de género en donde se demuestra y confronta constantemente el pensar de ser hombre (Gómez, 2020).

De este modo, la familia tiene un papel principal en la influencia y consolidación de la masculinidad a través del acompañamiento y el ejercicio de transmisión de saberes generacionales del comportamiento del hombre en la sociedad (Cyrulnik, 2020). En donde el 90\% reconoce que la familia como primera red influye de manera significativa en establecer la identidad del hombre en cuanto a la personalidad y comportamiento.

[...] La familia y las enseñanzas ya sea impuestas, educativas o tradicionales terminan por convertirse en un adoctrinamiento de las personas al estar expuestas de manera constante a dicha información y al compartir de manera habitual en un medio familiar y social especifico. (Camilo, comunicación personal, 2020)

A partir de lo anterior, se plantea que en la dinámica familiar en relación a la identidad del hombre se relaciona a procesos relacionados a las interacciones sociales mediados por patrones culturales aceptados reflejando la prevalencia de estereotipos masculinos afectados por los diferentes cambios sociales (Reyes, Díaz \& Vázquez, 2020). Esto plantea en la familia que la 
masculinidad se asocia a la asignación de roles y funciones que enmarcan el comportamiento del hombre planteado en la aceptación de su identidad constituyéndose en escenario de reproducción de roles, patrones y normas (Albuquerque \& Schraiber, 2020).

Por lo cual, se puede constatar que el $95 \%$ del proceso de identidad del hombre en el contexto familiar parte de las dinámicas y relaciones entre padre e hijos y en las conductas aprendidas que se dan por medio de la comunicación (Botero, Montoya \& Ospina, 2019). En donde la familia es fundamental para la construcción de nuevos referentes masculinos que configuran la identidad y los significados de la masculinidad asociados a modelos tradicionales.

[...]Pues en mi casa todos somos relativamente iguales, aquí no es que hay el hombre hace tal cosa o la mujer tiene que ser otra ¡no! todos nos colaboramos en los quehaceres, entonces no es tanto el decir. Aunque al momento de hacer fuerza siempre es el hombre el que la hace, ósea trabajos fuertes siemel pre lo hace el hombre de la casa, pero aquí a todos nos enseñaron lo mismo, a cocinar, a arreglar la casa, entonces no, es como tal que el hombre tiene que hacer esto y la mujer esto sino aquí todos hacemos por igual. (Marcos, comunicación personal, 2020)

A partir de lo anterior, la identidad se relaciona a la expresión del género que se valida en el ámbito de la familia soportado en funciones y roles asignados y establecidos socialmente que consolida la visión binaria de hombre-mujer (Botero, Caicedo \& Hernández, 2019). Por ejemplo, la asignación del color de acuerdo al género azul a niño y rosa a niña, o la selección de juguetes muñecas a niña y carros a niño, acciones que experimentan y aprenden en la percepción de identidad asociada la género y que plantea la reflexión de cambio en la construcción de masculinidad.

Por tanto, el 75\% de los hombres consideran que han copiado conductas de sus padres como son el tomar alcohol, jugar ciertos deportes $y$ 
comportamientos en el hogar planteando la familia como factor relevante para comprender la identidad masculina (Cruz \& Ospina, 2020). Finalmente, la familia es el escenario donde se comunica y expresa la referencia al género a través de comportamientos, vestimenta, relaciones, patrones, roles y vínculos la identidad de la persona para el planteamiento de normas sobre masculinidad (Gómez, 2020).

Por otro lado, un $65 \%$ de los hombres consideran que en el contexto social cercano es de influencia en la construcción de la identidad ya que reafirma patrones y roles comunes para el hombre que se afianzan a través de la presión de pares y la necesidad de ser parte de un grupo social (Jiménez \& Galeano, 2020). Sin embargo, es importante señalar los hombres no tienen las mismas experiencias frente a la construcción de identidad ya que hay diferentes aspectos como la edad, la condición socioeconómica, los roles y estatus social que influye en la concepción de masculinidad (Iturra, 2003).

En la mayor parte de los casos cada hombre tiene determinadas características biológicas, físicas y sociales en función de la asignación de roles que son aceptados socialmente evidenciando que al referirse al concepto de masculinidad lo relacionaban en un $68 \%$ al comportamiento agresivo y violento del hombre y un $32 \%$ lo relacionan más a aspectos biológicos y físicos. Por tanto, es importante señalar que el contexto social genera procesos de identidad del hombre a partir de estereotipos determinantes para los rasgos, actitudes, valores y características que se deben asumir planteando la necesidad de una reflexión más amplia de lo masculino (Llumipanta, 2018).

[...]Ummm pues ósea no consideró como tal que hay deportes que sólo se pueden practicar hombres o mujeres sino estéticamente para mí concepto si hay deportes que se ven mejor en hombres o mujeres, pero no a mí eso no. Como por ejemplo la natación artística y el fútbol americano, es un deporte para mí muy brusco para que lo practiquen mujeres, pero no es algo tan relevante (Edwin, comunicación personal, 2020). 
Por tanto, es posible afirmar que la construcción de identidad del hombre en el contexto social resulta de la relación de imaginarios y habilidades afrontadas al planteamiento de nuevas formas de percepción de la masculinidad (Jiménez \& Galeano, 2020). Sin embargo, comprender las nuevas identidades de la masculinidad en el ámbito social se presenta en situaciones de discriminación y temor en la expresión de emociones en donde el 75\% de los hombres se sienten intimidados en expresar la emoción de tristeza en público y el $25 \%$ se siente intimidado si socialmente expresa la ternura y lo asocia a debilidad.

Finalmente, la identidad de lo masculino en el ámbito social se da como proceso mediado por prácticas y habilidades atribuidas al ser hombre como la fuerza, que incide en la necesidad de aceptación social a partir de valores que construyen estereotipos ideales del hombre (Ballén, 2019). De este modo, se puede sustentar la crisis de identidad del hombre en la necesidad de establecer habilidades sociales que exploren, establezcan y comprendan las nuevas formas de construcción de la masculinidad (Romero, 2018).

\section{CONCLUSIONES}

La globalización social desarrolla nuevas desigualdades sociales en el ámbito económico, cultural y político, lo cual representa nuevos desafíos para el ejercicio de la masculinidad que plantean nuevos retos al hombre (Muñoz, 2017); dadas por el consenso liberal, en donde el concepto de genero sustituye un abanico de posibilidades al ciudadano y el criterio de inclusión deja de ser el derecho, para pasar a ser la solvencia; en donde, el ser hombre es un desafío a nuevas necesidades del mundo (Pineda \& Otros, 2019).

Pensar en los hombres en el marco global, supone en los Estados proponer el concepto de regulación de vulnerabilidades y riesgos diferenciales en los actores sociales a partir de instrumentar una estrategia integral de programas 
(Torns \& Recio, 2012). Aspecto que genera una crisis debido a la creciente fragmentación social, la pobreza, el desplazamiento, la crisis creciente de la justicia y la corrupción de las instituciones (Moreno, 2017).

Entonces, si las instituciones son una posibilidad o actor clave en la construcciones de identidad puede disminuir la crisis en la perspectiva de género trazando una línea de reconocimiento de nuevas formas de concebir al hombre (Lopes, 2019). Esta ya no se presenta hoy como una frontera fija que divide dos zonas claramente separadas el ser hombre o mujer; sino, que es una línea movediza tras de la cual quedan situadas en zonas más y más amplias de la vida social (Lagarde, 2018). Por tanto, el hombre al no sentirse reconocido desde sus emociones sino como una construcción social aporta a la crisis de una identidad pública clara (Martínez, 2019).

En ese sentido, una identidad como intento de justificar el diseño de intervenciones para los hombres, gira entorno a la discusión sobre capital humano en la relación entre productividad y progreso técnico, como variables relevantes en la identidad de lo público (Gomes, 2018). De la misma forma, es importante la reflexión y el papel del Estado, ya que refleja múltiples enfoques y análisis del papel de la identidad de los hombres en relación con la consideración política, económica, social y cultural del género (Connel \& Pearse, 2018).

En esta perspectiva de género en el contexto social tiende a ser coherentes a las necesidades de estereotipos del género para el establecimiento de roles y funciones (Cabezas, 2019). Por tanto, se estructuran programas que quieren optimizar los servicios y dar soporte a las necesidades de una sociedad, pero no a las necesidades de los hombres, premisa que plantea conceptualizar de manera compleja la percepción del hombre más allá del sujeto fuerte y de poder que se forma en el contexto familiar (Bedia, 2005). 
Planteándose una reflexión en la manera en cómo se reconstruye la entidad del hombre de manera sistemática en contextos como el medio ambiente, la familia, la comunidad que permiten evidenciar patrones que dan respuestas a las necesidades de los hombres en la relación salud-enfermedad, social, económico, cultural (Ballén, 2019). Sin embargo, en el momento de implementar los diferentes modelos de comprensión del hombre en un contexto como la familia se plantea en la realidad problemas en las acciones del Estado, donde la implementación de modelos no son acordes a sus necesidades o las realidades sociales contemporáneas de los hombres (Cruz \& Ospina, 2020).

Es importante que la gama de opciones disponibles y los logros que se alcanzan al interior de las iniciativas de las mujeres aumenten o disminuyan con relativa independencia del rol del hombre en función de variables culturales o distributivas de la capacidad de una sociedad para proporcionar bienes públicos como la seguridad o en este caso la identidad que en lo general el hombre debe proveer (Cabezas, 2019). Entonces, el capital humano desde una intervención institucional para los hombres puede estar relacionado con el modelo neoliberal, en el papel que desarrollan las instituciones en la relación de los factores de poder que van dirigidos a un proyecto de eficiencia soportado en lo económico, en un contexto que muestra una grave situación de pobreza y una corrupción generalizada esto puede producir un desencanto o quiebre en el fortalecimiento de la identidad del ser hombre (Bautista \& Barquín, 2018).

Finalmente, es importante analizar los imaginarios de identidad que tienen los hombres, en la dimensión del rol en el contexto familiar y social, ya que puede generar una identidad masculina y un capital humano diferente a la percepción tradicional (Gómez, 2020), con la capacidad de vincularse a las 
dinámicas del mercado, a partir de las políticas públicas, estas entendidas como la suma de las actividades del gobierno bien sea directamente o a través de agentes, las cuales tienen influencia en la vida de los ciudadanos (Donat \& Díaz, 2013).

Una vez analizada la información recolectada a través del presente estudio, se pude señalar como conclusión que las representaciones sociales en cuanto a la identidad masculina inciden en la posibilidad de comprender los comportamientos del hombre a partir de las formas de comunicación y el lenguaje que facilitan la repetición de actitudes hegemónicas de la masculinidad. Planteando la necesidad de incidir no solo en reformar la formación de la sociedad en temas de masculinidad sino también en la necesidad de reivindicar al hombre desde la manifestación abierta de emociones

Por tanto, poder analizar las representaciones sociales de la masculinidad es importante plantearlo desde la familia como uno de los escenarios más influyentes en la transmisión de patrones culturales en esta reflexión, y que sería necesario que se observará como punto de partida en la construcción de cambios en la identidad del hombre frente a procesos sociales diferentes donde se asumen nuevos roles de autocuidado, protección y de manifestación de emociones, para poder fomentar nuevas formas de la masculinidad.

Finalmente, como prospección de futuro, se plantea continuar con el desarrollo de iniciativas de investigación que relacionen la necesidad de analizar las dinámicas de las masculinidades en escenarios locales que enriquezcan la identidad del hombre. De este modo se pueda superar prejuicios y estereotipos de la masculinidad a partir de la exploración, descripción e interpretación de las nuevas formas de concebir la masculinidad. 


\section{REFERENCIAS}

Albelda, J. S. (2018). El cuerpo masculino en tiempos de brújulas rotas y (neo) fascismos: análisis socioantropológico. Revista Nuevas Tendencias en Antropología, (9), 15-33. http://www.revistadeantropologia.es/Textos/N9/El\%20cuerpo\%20m asculino\%20en $\% 20$ tiempos\%20de.pdf

Albuquerque, F. P. D., \& Schraiber, L. B. (2020). Masculinidad y fútbol: cuestiones de género en una experiencia de rehabilitación psicosocial de hombres en el Distrito Federal, Brasil. Salud Colectiva, 16, e2247. https://doi.org/10.18294/sc.2020.2247

Alcántara, E. (2013). Identidad sexual/rol de género. Debate feminista, 47, 172-201. https://www.jstor.org/stable/43832471

Bard Wigdor, G. (2018). Las violencias romantizadas: masculinidades hegemónicas en el capitalismo tardío y heteropatriarcal. Apostadigital; $\quad$ Aposta; $\quad 77 ; \quad 6-\quad 2018 ; \quad 59 \quad-100$. http://apostadigital.com/revistav3/hemeroteca/gbard.pdf

Bautista, G. V., \& Barquín, H. S. (2018). Violencia de género, masculinidades y políticas públicas. Temas sociológicos, (22), 151-176. https://dialnet.unirioja.es/servlet/articulo?codigo $=6780098$

Ballén Gavilánez, F. A. (2019). Construcción de estereotipos masculinos a partir de la percepción sobre personajes cinematográficos en las estudiantes de octavo semestre de la Facultad de Comunicación Social de la Universidad de Guayaquil, 2018. Casos: Christian Grey, Steve Rogers y Thor (Bachelor's thesis, Universidad de Guayaquil, Facultad de Comunicación Social).

Bedia, R. C. (2005). El género en las ciencias sociales. Cuadernos de trabajo social, 18, 249-258. 
https://revistas.ucm.es/index.php/CUTS/article/view/CUTS0505110 249A

Botero, L. D. B., Montoya, J. C., \& Ospina, J. C. H. (2019). Configuración de la identidad en padres de familia adolescentes. Revista Colombiana de Ciencias $\quad$ Sociales, 10(2), 377-398. https://dialnet.unirioja.es/servlet/articulo?codigo $=7028117$

Butler, J. (2004). Lenguaje, poder e identidad. Editorial Síntesis.

Cabezas, S. (2019). Masculinidad, violencia de género y políticas públicas. Tesis licenciatura Trabajo Social. Universidad de la república, facultad de ciencias sociales, Departamento de Trabajo Social.

Cyrulnik, B. (2020). El murmullo de los fantasmas: volver a la vida después de un trauma. Editorial Gedisa.

Connel, R., \& Pearse, R. (2018). Género: desde una perspectiva global. Universitat de València.

Cruz García, D. A., \& Ospina, A. M. L. (2020). El hombre como expresión de identidad en el contexto contemporáneo. Centro Sur, 4(1). https://doi.org/10.37955/cs.v4i1.48

Del Río Almagro, A., \& Pastrana de la Flor, M.M. (2018). Las estrategias artísticas ante el cuestionamiento de la masculinidad hegemónica en la sociedad occidental: De la crisis de finales del siglo XX a su resurgimiento en la actualidad. Masculinities and Social Change, 7(2), 153-177. http://dx.doi.org/10.17583/MCS.2018.2813

Donat, Julián A., \& Díaz, I. B. (2013). Estereotipos y prejuicios de género: Factores determinantes en Salud Mental. Norte de salud mental, 11(46), 20-28. https://dialnet.unirioja.es/servlet/articulo? codigo $=4694952$

Gallego-Montes, G. (2018). Estudios de familia en clave de masculinidades. Estado de la discusión en Colombia. Revista Latinoamercana de 
Estudios de $\quad$ Familia, 10(2), 30-50. http://vip.ucaldas.edu.co/revlatinofamilia/downloads/Rlef10(2)_3.pd $\mathrm{f}$

Gómez Machado, A. M. (2020). Representaciones sociales de la masculinidad en la narrativa de Grand Theft Auto V. http://hdl.handle.net/20.500.12209/12391

Gómez Beltrán, I. (2019). Grindr y la masculinidad hegemónica: aproximación comparativa al rechazo de la feminidad. Estudios sociológicos, 37(109), $39-68$. http://www.scielo.org.mx/scielo.php?script=sci_serial\&pid=24486442

Gomes, C. D. M. (2018). Gênero como categoria de análise decolonial. Civitas-Revista de Ciências Sociais, 18(1), 65-82. https://doi.org/10.15448/1984-7289.2018.1.28209

Iturra, R. (2003). La construcción social de la masculinidad. In Hombres: La construcción cultural de las masculinidades (pp. 25-52). Talasa. https://dialnet.unirioja.es/servlet/articulo?codigo $=837822$

Izquierdo, D. (2010). El juego de rol como instrumento psicopedagógico para la exploración y comprensión de la identidad sexual y de género. Tándem: Didáctica de la educación física, (34), 64-74. https://dialnet.unirioja.es/servlet/articulo?codigo $=3276230$

Lagarde, M. (2018). Género y feminismo: desarrollo humano y democracia. Siglo XXI Editores México.

Lopes Louro, G. (2019). Currículo, género y sexualidad. Lo" normal", lo" diferente" $\quad$ y $\quad$ lo" excéntrico". Descentrada, 3. https://doi.org/10.24215/25457284e065 
Llumipanta Panchi, L. E. (2018). Lo masculino"¿ estereotipo o construcción social?. Un análisis crítico de los enfoques de trabajo sobre nuevas masculinidades (Bachelor's thesis, Quito: UCE).

Martel Díaz, A. L. (2017). Representaciones sociales: Neo machismo y Neo sexismo en ciencia y tecnología, participación de los estudiantes millennials de la Universidad Nacional de San Agustín de AREQUIPA-2017.

Martínez, A. (2019). La crisis del héroe: una autoetnografía sobre la pérdida de la masculinidad hegemónica. https://repositorio.uesiglo21.edu.ar/handle/ues21/17435

Martínez, N. G. M., Espinosa, M. L. I., Landgrave, G. C., \& Ruiz, E. J. C. (2018). La sexualidad en adolescentes desde la teoría de las representaciones sociales. Psicología y salud, 28(1), 15-24.

Mio Torres, L. E. (2018). Representaciones sociales sobre la masculinidady su relación con la violencia de pareja según jóvenes universitarios. Universidad Antonio Ruiz de Montoya. http://repositorio.uarm.edu.pe/handle/UNIARM/1936

Moncayo Saltos, K. D., \& Rodríguez Rosario, A. J. (2019). Representaciones sociales de género, conocimientos, actitudes y prácticas sobre salud sexual en estudiantes de primer nivel de la Pontificia Universidad Católica del Ecuador 2018. Tesis especialista familiar y comunitaria. Pontificia Universidad Católica del Ecuador. http://repositorio.puce.edu.ec/handle/22000/16914

Montenegro, J. L., Orcasita, L. T., Tunubala, L. A., \& Zapata, L. J. (2019). Representaciones sociales sobre masculinidad y paternidad en padres con hijos gays. Investigación en Enfermería: Imagen $y$ Desarrollo, 21(1). https://doi.org/10.11144/Javeriana.ie21-1.rsmp 
Moreno-Seco, M. (2017). Cruce de identidades: masculinidad, feminidad, religión, clase y juventud en la JOC de los años sesenta. Historia y Política. 2017, 37: 147-176. https://doi.org/10.18042/hp.37.06

Muñoz, L. S. (2018). Relaciones de género y arreglos domésticos: Masculinidades cambiantes en Concepción, Chile. Polis. Revista Latinoamericana, (50). https://journals.openedition.org/polis/15742

Muñoz Sánchez, H. (2017). Hacerse hombres: La construcción de masculinidades desde las subjetividades. Fondo Editorial FCSH.

Newman, G. D. (2006). El razonamiento inductivo y deductivo dentro del proceso investigativo en ciencias experimentales y sociales. Laurus, 12(Ext), 180-205. https://www.redalyc.org/pdf/761/76109911.pdf

Núñez Noriega, G., \& Espinoza Cid, C. E. (2017). El narcotráfico como dispositivo de poder sexo-genérico: crimen organizado, masculinidad y teoría queer. Revista interdisciplinaria de estudios de género de El Colegio de México, 3(5), 90-128. https://doi.org/10.24201/eg.v3i5.119

Pérez, M., \& Radi, B. (2018). El concepto de violencia de género como espejismo hermenéutico. Igualdad, autonomía personal y derechos sociales, 8, 69-88. https://www.aacademica.org/blas.radi/36

Pineda-Roa, C. A., Galindo-Ascanio, G. K., González-Moreno, D. P., \& Chaparro-Clavijo, R. A. (2019). Validación de un inventario de roles de masculinidad hegemónica en varones colombianos. Diversitas (17949998), 15(2). https://doi.org/10.15332/22563067.5401

Rivera, D. C. L., \& de los Santos, P. J. (2017). Representaciones Sociales sobre las personas LGBTI en la universidad: perspectivas del profesorado y alumnado. Revista de Educación Inclusiva, 9(3). https://revistaeducacioninclusiva.es/index.php/REI/article/view/264 
Romero, R. B., \& Gea, E. G. (2019, November). EL DISCURSO VISUAL DEL ETERNO MASCULINO EN TANGANA. ROLES DE MASCULINIDAD ENTIEMPOS FEMINISTAS. In I Congreso internacional sobre masculinidades e igualdad: en busca de buenas prácticas de masculinidades igualitarias desde el ámbito de la Universidad (p. 40). Universidad Miguel Hernández.

Romero Miranda, A. (2018). Construcción de masculinidad y belleza masculina en jóvenes varones infractores de ley consumidores problemáticos de drogas. Ultima década, 26(48), 107-132.

http://dx.doi.org/10.4067/S0718-22362018000100107

Rubinacci, D. (2019). Las raíces de la violencia del hombre contra la mujer: reflexión sobre la construcción de la masculinidad. Journal of Psychosocial Systems, 3(1), $13-24$. https://doi.org/10.23823/jps.v3i1.50

Salinas, S. C., \& Núñez, J. M. J. (2019). Las interculturalidad-es, identidades y el diálogo de saberes. Reencuentro. Análisis de problemas universitarios, (66), $10-23$. https://reencuentro.xoc.uam.mx/index.php/reencuentro/article/view/8 21

Sánchez, F. L. (1988). Capítulo 2: Adquisición y desarrollo de la identidad sexual y de género. In Nuevas perspectivas en el desarrollo del sexo y el género (pp. 47-69).

Torns, T., \& Recio, C. (2012). Las desigualdades de género en el mercado de trabajo: entre la continuidad y la transformación. Revista de economía crítica, 14(segundo semestre), 178-202. http://revistaeconomiacritica.org/sites/default/files/revistas/n14/Semi monografico-4.-Torns-Recio.pdf 
Tubay, F. M. (2019). Estereotipos de género: Perspectivas en profesiones artesanales de Portugal. Revista Estudos Feministas, 27(2). https://doi.org/10.1590/1806-9584-2019v27n254182

Viveros Vigoya, M. (2018). Las mujeres en la investigación sobre masculinidad-Antología del pensamiento Social en Colombia. $\begin{array}{llll}\text { Universidad Santiago de } & \text { Cali. }\end{array}$ https://repository.usc.edu.co/handle/20.500.12421/1279

Winfield Reyes, A. M., Jiménez Galán, Y. I., \& Topete Barrera, C. (2017). Representaciones mentales y sociales en la equidad de género. $L a$ ventana. Revista de estudios de género, 5(45), 186-210. http://www.scielo.org.mx/scielo.php?script=sci_arttext\&pid=S140594362017000100186 